\title{
Time slows down during accidents
}

\author{
Valtteri Arstila * \\ Department of Behavioral Sciences and Philosophy, University of Turku, Turku, Finland
}

\section{Edited by:}

Bill Mace, Trinity College, USA

\section{Reviewed by:}

Bill Mace, Trinity College, USA

Tomer Fekete, SUNY Stony Brook, USA

\section{${ }^{*}$ Correspondence:}

Valtteri Arstila, Department of

Behavioral Sciences and Philosophy,

University of Turku, 20014 Turku,

Finland.

e-mail: valtteri.arstila@utu.fi
The experienced speed of the passage of time is not constant as time can seem to fly or slow down depending on the circumstances we are in. Anecdotally accidents and other frightening events are extreme examples of the latter; people who have survived accidents often report altered phenomenology including how everything appeared to happen in slow motion. While the experienced phenomenology has been investigated, there are no explanations about how one can have these experiences. Instead, the only recently discussed explanation suggests that the anecdotal phenomenology is due to memory effects and hence not really experienced during the accidents. The purpose of this article is (i) to reintroduce the currently forgotten comprehensively altered phenomenology that some people experience during the accidents, (ii) to explain why the recent experiments fail to address the issue at hand, and (iii) to suggest a new framework to explain what happens when people report having experiences of time slowing down in these cases. According to the suggested framework, our cognitive processes become rapidly enhanced. As a result, the relation between the temporal properties of events in the external world and in internal states becomes distorted with the consequence of external world appearing to slow down. That is, the presented solution is a realist one in a sense that it maintains that sometimes people really do have experiences of time slowing down.

Keywords: perception, consciousness, the passage of time, phenomenology, temporal illusions

\section{INTRODUCTION}

This paper focuses on the experiences that people anecdotally have had during frightening events and especially on how to account for the altered phenomenology in those events. The main interest lies on the experience of time slowing down, which is the most frequently reported case of altered phenomenology during the accidents. This temporal distortion is a fundamental one because it concerns our sense of time passing when experiences flow from one to another, which is at the very heart of the stream of consciousness, and it concerns the perception of all stimuli alike, not merely some particular sensory system.

In more detail, the paper has three interrelated objectives. The first one is to describe some of the relevant features of those experiences and situations where people have claimed that time slowed down for them. Understanding both the phenomenology and the situation where such distortions (allegedly) occur is important, for the experiences people report having during accidents are not confined to the phenomenology of time slowing. Hence, time distortions do not constitute the whole explanandum.

The second objective is to argue that the described phenomenology really occurs during accidents. This will be done by showing how the two recently presented alternative explanations of the phenomenon, both presented in Stetson et al. (2007), are unsatisfactory. The issue at stake here is important for the reason that it is intimately tied to the question whether the speed of the passage of time, the flow of the stream of consciousness, is ever really experienced. Presumably, if it is not experienced even in these often-referred anecdotal cases, then it becomes doubtful whether it is experienced in normal situations either. This result, in turn, would have significant consequences for the theories of time consciousness, many of which posit that we have the experiences of the passage of time (for old but still relevant theories, see Husserl, 1991; James, 1890, for more recent discussions see Dainton, 2000; Lloyd, 2002).

Finally, after arguing that there are good reasons to accept peoples' reports of time slowing down during accidents, the third objective is to provide a phenomenally and empirically plausible account of the described experiences. To this end, the shortcomings of the discussed theories are also useful as they point toward a possible solution that will be presented in the final section. There is indeed a need for such theory, since apart from the rejected explanation and (possibly) related explanations concerning performances in various duration estimation tasks, we currently have no theory to account for the phenomenology of these experiences. In short, the proposed theory is that the experiences of time slowing down are related to the increased speed of internal processes: when we become faster, the world appears slower to us. It is furthermore hypothesized that our faster internal processes are due to locus coeruleus norepinephrine system, whose activity increases in frightening accidents, and that our sense of normal speed of external events adapts to the prevailing conditions, which is why we have experiences of the time slowing down only in unusual circumstances.

\section{THE PHENOMENOLOGY OF TIME SLOWING DOWN}

The first detailed characterization of the experiences in question comes from geologist Albert von St. Gallen Heim (translated in Noyes and Kletti, 1972). In the Yearbook of the Swiss Alpine Club 1892 , Heim wrote that nearly $95 \%$ of climbers, who reported their 
experiences during an accidental fall to him, had had an experience that in part included

a dominant mental quickness and sense of surety. Mental activity became enormous, rising to a 100 -fold velocity or intensity. The relationships of events and their probable outcomes were overviewed with objective clarity. No confusion entered at all. Time became greatly expanded. The individual acted with lightning-quickness in accord with accurate judgment of his situation. (Noyes and Kletti, 1972,pp. 46-47)

The next descriptive, and more systematic, analyses by Noyes and Kletti $(1976,1977)$ basically confirmed Heim's claims. The most frequently reported features in the survey were an apparent slowing down of external time (75\% of 85 participants reported this in the 1976 study, $72 \%$ of 101 participants in the 1977 study) ${ }^{1}$. Almost as many subjects reported an increased speed of thoughts (68 vs. 61\%). A 14-year-old boy who had accidentally shot himself in the chest, for example, reported that his "thoughts were speeded up and time seemed stretched out" (Noyes and Kletti, 1976, p. 22). For some, the experience of time slowing down was even so strong that "time stood still" (Noyes and Kletti, 1976, p. 21). As a summary of these two features, they write:

Not only did elapsed time seem drawn out, but events seemed to happen in slow motion. Yet in contrast to the outward slowing, individuals described their thoughts as speeded up and expressed amazement at the number of thoughts or mental images that passed through their minds in a matter of seconds. These two aspects of the experience of time were generally described together and were clearly related to one another. (Noyes and Kletti, 1976, p. 23)

Furthermore, many of Noyes and Kletti's subjects reported having acted fast and purposefully combined with increased focused attention and alertness that were also present in Heim's characterization. For instance, a 19-year-old climber reported that during his fall, the fear and hope of survival "forced a concentration of my thoughts on rescue efforts and a redirection of my whole mind onto whatever might be necessary to prevent the potential plunge" (Noyes and Kletti, 1976, p. 23). Similarly, a jet pilot during the Vietnam War showed the same increased attention and alertness to actions needed to save his life when his jet was improperly launched:

... when the nose-wheel strut collapsed I vividly recalled, in a matter of about $3 \mathrm{~s}$, over a dozen actions necessary to successful recovery of flight attitude. The procedures I needed were readily available. I had almost total recall and felt in complete

\footnotetext{
${ }^{1}$ It should be mentioned that the phenomenology that St. Gallen Heim, Noyes, and Kletti describe is similar to that reported in near-death experience studies. Greyson and Stevenson (1980) for example found that $79 \%$ of the respondents in the neardeath study had had anomalous temporal phenomenology. Direct comparison of the studies is impossible, however, for two reasons. First, near-death studies are often less explicit about the altered phenomenology in general and focus more on changes on attitudes and religious experiences. Second, the subject populations that provide the reports are very different. For example, while the subjects in Noyes and Kletti's studies had been conscious during the experiences, $40 \%$ of Greyson and Stevenson subjects had been on such a bad condition that they had been considered dead during the near-death experience.
}

control. I seemed to be doing everything that I could and doing it properly. (Noyes and Kletti, 1976, p. 24)

To summarize, the key features of the experiences that are of interest here are the following:

1. The feeling of external time expanding and slowing down to a great extent.

2. Dominant mental quickness as demonstrated by the increased speed of thoughts.

3. There is often an altered sense of the duration of the event lasting longer than it actually does.

4. If possible, in the event in question, people often act fast and purposefully.

5. In the latter case, their attention is also altered and narrowly focused on the issues relevant for survival.

6. Unusually sharp vision or hearing.

It is important to notice that experiences having these features occur only in exceptional situations because this kind of experience is a "normal reaction to suddenly presented, life-threatening danger" (Noyes and Kletti, 1976, p. 26). Thus one relevant factor for triggering these experiences appears to be the belief in imminent death - the experiences are more probable if a subject believes that she is in a fatal situation. Indeed, in Noyes and Kletti's (1976) survey, $80 \%$ of the subjects $(N=59)$ who believed that death was imminent reported experiences of time slowing down, whereas such experience was reported only by $65 \%$ of the subjects $(N=26)$ who did not believe death was imminent [in 1977 study, the percentages were $78(N=59)$ and $63(N=42)]$. An even more important factor is that the event is surprising, since Noyes and Kletti argue that hospital patients facing a threatening situation due to their illness do not report an increased speed of thoughts and altered attention. Heim, in turn, also referred to both factors as he mentioned that people who have had these experiences can be completely paralyzed in less surprising and/or less dangerous situations. Given the phenomenology of the experiences and the knowledge of the typical situations in which they can occur, how can we investigate and explain these experiences?

\section{RECENT INVESTIGATION OF THE EXPERIENCES DURING FRIGHTENING EVENTS}

Two recent and different lines of research concern temporal features of the phenomenology in question. One of them relates to the first listed key feature (the feeling of external time expanding), whereas the other relates to the third feature (the altered sense of duration). While the first feature is the main topic of this paper, let us begin with the latter line of research. One motivation to do so is to clarify the difference between the two features. There is need for such because the first and third key features of the phenomenology both concern temporal phenomena and the expression "time slows down" has been used in relation to both of them. Accordingly, one might think that these two features boil down to the same phenomenon in the final analysis. Yet, a closer inspection of the both features illustrates that the expression "time slows down" has a different meaning in these two contexts and that such conclusion is unwarranted. 
The first key feature concerns the subjective feeling of the passage of time - whether it goes fast or slow. Here the feeling of time slowing down could be, for example, a consequence of a distorted phenomenology: when the movement we perceive happens in slow motion, we have the feeling that time slows down. When reading the subjective reports, this expansion of time appears to be quite substantial for subjects - a few seconds, might for example feel like 5 min (Noyes and Kletti, 1977, p. 376). Although such reports are common, literally taken this 100 -fold expansion of time is rather unbelievable. Hence some skepticism toward such reports is warranted. This does not mean that they should be merely dismissed though. Rather, they should be taken as a sign of such remarkable change in the phenomenology that one has difficulties in making duration estimations. In this paper, the expression "time slows down" is used in this sense that refers to changes in one's phenomenology of time passing by ${ }^{2}$.

The third key feature concerns the explicit cognition of the duration of passed time. It concerns duration estimation (also called duration or interval perception) - our performance in various tasks such as prospective and retrospective interval estimation tasks, interval production and reproduction tasks, and temporal bisection tasks. In these studies, "time slowing down" refers to the overestimation of the duration of stimuli.

Time slowing down understood in this sense is often explained by the means of an (hypothetical) internal clock (e.g., Gibbon, 1977; Treisman et al., 1990; Wearden, 1991). The idea is that when the speed of this clock is increased, more ticks occur during the measured interval. As more ticks means longer duration, subjects overestimate the duration in question. In such cases, subjects may estimate that something lasted, say, $5 \mathrm{~s}$ although in reality it lasted only $4 \mathrm{~s}-\mathrm{a} 4 \mathrm{~s}$ interval slowed down in a sense to a $5 \mathrm{~s}$ interval.

While there has been little empirical research on the phenomenology of time passing by, there is a considerable amount of research on our performance in various duration estimation tasks. Thus, if the two usages of "time slows down" would be the same, or if they would be different sides of the same phenomenon (one focusing the phenomenology and the other concerning performance in tasks), there would in fact be a number of studies on the matter already. For example, it has been found that fear is associated with time slowing down in duration estimation studies (Campbell and Bryant, 2007) and that threat causes subjects to overestimate durations (e.g., Anderson et al., 2007). Furthermore, some of the studies on performance in time perception tasks using pictures or video of a threatening situation even use the expression "time slows down" in their titles (Loftus et al., 1987; Bar-Haim et al., 2010).

\footnotetext{
${ }^{2}$ This usage is close to the frustrating feeling that time moves slowly, drags, when we are bored. The phenomena that are behind these two are different however. One difference between these two phenomena is that in the latter case the feeling of time dragging, moving slowly, evolves slowly whereas it happens almost immediately in the phenomenon we are interested in. Second, when we are bored we do not really experience time slowing down in a sense that things move in slow motion, whereas this is what people report they experience during accidents. Third, when people are bored, they still do not report any altered (or at least increased) speed of thoughts and attention. Accordingly, there are good reasons to separate the experience of time moving slowly when we are bored from the phenomenon discussed here.
}

Despite these possible similarities, the studies on the performance in duration estimation tasks need to be separated from the passage of time judgments and therefore they can provide only little help in addressing the topic of this paper. To begin with, our duration estimations and the passage of time judgments can change independently of each other (Wearden, 2008). Hence duration estimations can be at best only indirect measures of the phenomenology of time passing by. Stated more generally, because the mentioned studies only concern our performance on the duration estimation tasks, they do not address the issue of the distorted phenomenology and consequently can only (at best) provide us information on the third key feature, leaving others unanalyzed. This conclusion is emphasized by the fact that the duration overestimation results are explained by means of an internal clock without any reference to the phenomenology of time slowing down and other distorted phenomenology. Thus it remains an open question why speeding of an internal clock would bring about, say, an unusually sharp hearing.

Second, the two phenomena are often of different magnitude. In duration estimation studies, the idea that time slows down follows from the fact that the duration of stimuli is overestimated often by $10-50 \%$. In the cases that Noyes and Kletti describe, time appears to slow down a 100 -fold. This difference in magnitude suggests that the internal clock metaphor cannot be the sole explanation for time slowing down in the phenomenological sense used in this paper.

Finally, it is not obvious how much we can justifiably conclude even from the duration estimation studies mentioned above that appear to be most related to the topic at hand - after all they investigated time slowing down in response to threatening stimuli. One reason for skepticism is that the duration to be judged was much longer than in those that Noyes and Kletti describe ranging from a half minute (Loftus et al., 1987) to half an hour (Campbell and Bryant, 2007), which in turn makes it likely that they investigate somewhat different phenomena. Likewise, in these studies the stimuli were presented many times and under controlled conditions. Therefore, the situations were unlikely to match with Noyes and Kletti's observation that their explanandum occurred in surprising and life-threatening situations.

Taken together, the listed reasons make it safe to say that the studies on the performance in duration estimation tasks cannot shed much light on the issues at hand, even if there is a connection, possibly a close one, between them and the subjective feeling of the passage of time. Accordingly, the vast amount of research on duration estimation, even when it relates to threatening situations, will be largely ignored here.

There is, however, a recent study by Stetson et al. (2007) that is more directly pertinent to the issues at hand. In this study, the idea of people experiencing that time slows down during a frightening event is introduced with a motion picture analogy: when a movie runs slower than it was filmed, things in it appear to happen slowly. What makes this idea tempting is that it readily fits with the reports of how time in the external world appears to slow down and thus the authors' approach to time slowing down is the same as the phenomenological one used here. One of Noyes and Kletti's subjects, who narrowly escaped a collision with a train, for example said that "as the train went by I saw the engineer's face. 
It was like a movie run slowly so the frames progress with a jerky motion. That was how I saw his face" (Noyes and Kletti, 1977, p. 377). In line with such reports, Stetson et al. (2007) argue that the slow motion of time "should entail consequences such as an ability to perceive things with higher temporal resolution. For example, watching a hummingbird in video slow motion allows finer temporal discrimination upon replay at normal speed because more snapshots are taken of the rapidly beating wings."

To test this hypothesis, the authors conducted an experiment where subjects' temporal resolution was measured under normal and frightening conditions. The normal condition here refers to casual non-frightening conditions whereas in the frightening condition the subjects "were released from the top of the tower and experienced free fall for $2.49 \mathrm{~s}$ before landing safely in a net" (Stetson et al., 2007). The threshold for temporal resolution in turn was measured with two flickering stimuli (a digit and its negative image) and the subjects' task was to separate them from each other in both conditions. What they found was that the subjects' temporal resolution did not improve when they faced a frightening situation. That is, the number of "snapshots" did not increase during the fall.

In addition to this task, some of the subjects were asked to estimate the duration of the fall twice. In the first time, they had seen someone else falling and they were then asked to estimate the fall's duration by imagining themselves being in the position of the falling person. The second estimation was based on the recollection of their own fall. What the authors found was that under the latter conditions the duration estimations were 36\% longer.

Based on their results, the authors concluded that although time appears to slow down during frightening events - the increase in the duration estimations illustrates how time becomes expanded similarly to what people report that they have experienced during accidents - this is not due to perception because the temporal resolution did not improve. Rather, they end their article by arguing that this is due to memory effects:

... we speculate that the involvement of the amygdala in emotional memory may lead to dilated duration judgments retrospectively, due to a richer, and perhaps secondary encoding of the memories. Upon later readout, such highly salient events may be erroneously interpreted to have spanned a greater period of time. (Stetson et al., 2007)

Stetson et al. (2007) put forward thus two competing explanations for the anecdotal phenomenology described above. According to the first one, the given reports are truthful and the external world does indeed appear to move in slow motion. This explanation was not supported by the evidence however. According to the second explanation, time slowed down only in the sense related to the duration estimation tasks. In this case one can wonder about the validity of the reports given after accidents. The authors' response is to acknowledge that such reports are indeed given but explain the unusual phenomenology by the means of unusual memory effects. Unfortunately, however, both of these explanations have their shortcomings: the first explanation and the temporal resolution task did not touch upon the issue they were supposed to, while the latter explanation cannot provide the full picture of the experiences during the frightening accidents. Or so I argue below.

\section{EXPLANATION BASED ON TEMPORAL RESOLUTION OF PERCEPTION}

What Stetson and colleagues tested was in effect the temporal resolution of visual perceptual processes - as separated, for example, from the issue of how many thoughts we can think in a second (i.e., are our thoughts speeded or not) - in controlled amusement park conditions. As such, there are a number of ways to argue that this study does not in fact look into the phenomenon it was supposed to. To begin with, there is a question whether the free fall can be counted as surprising or "suddenly presented, life-threatening danger" during which Heim, and Noyes and Kletti argue one can experience time slowing down. For example, all those participants that estimated the duration of the fall had seen the fall before they experienced it themselves and hence it is not obvious that this situation would count as surprising for them. If the situation was not surprising and time slowing down was not experienced, then what was studied is not what the authors claimed. A natural way to overcome this objection would be to ask participants directly whether time slowed down for them or not. Unfortunately the study did not report that this was done, and hence the question whether time did slow down remains uncertain.

One can of course argue that the difference in the duration estimations at least suggests that time did slow down, as the authors appear to think. In light of the earlier discussion on how these two can come apart, and the fact that the difference in the duration estimations was only one third (and it is thus of a very different scale than the subjects' reports of time expanding a 100-fold), it is obvious that this is only an assumption and that can easily be questioned. Furthermore, it is neither the only nor the most plausible explanation for the result of dilated duration estimation. For example, it has been reported that subjects underestimate the duration of a task they are about to do before they actually do it (e.g., Byram, 1997; Roy et al., 2005). As the duration of the fall was shorter in the task where subjects imagined the falling without having yet experienced it in the study, it could be that the difference simply reflects the type of tasks subjects carried out. In another experiment, where people imagined a second hand of the clock moving for 15 and $30 \mathrm{~s}$, the duration estimations were shorter when the clock is imagined to be farther away, i.e., when the image is smaller (Zäch and Brugger, 2008). This is in good accordance with the results that smaller stimuli are judged to last for a shorter duration than larger ones (Xuan et al., 2007). Given that in Stetson et al.'s study the subjects' perception of the situation took place from a distance (before they themselves were dropped), it could have influenced the subjects' performance when they were imagining the fall without actually having had the experience of it themselves yet. This provides another, empirically justified explanation for the results without relying on the idea that people experienced time slowing down. As a result, the possible lack of surprise and the existence of competing well-motivated explanations for the difference in the duration estimations together make the conclusions of the study unwarranted.

In keeping with the main objective of this paper, namely the question of how to account for the anomalous temporal experience during sudden and frightening situations, it is important to notice possibly even more fundamental problems with the study: the methodology and rationale behind it. As illustrated by the 
quotation above, Stetson et al.'s study is strongly influenced by the analogy to movies. Although this makes their rationale initially tempting, a closer consideration shows it to be problematic.

To begin with, even accepting that the difference in duration estimation is not due to the reasons discussed, the study only showed that the threshold for determining flickering stimuli and duration estimation are not related at least directly. If we accept Stetson et al.'s claim made in the course of their argumentation that subjective time is not a single unified entity, and there are good reasons to think that this is the case, then it is not obvious that the temporal resolution task the subjects did is suitable task to measure whether time slows down or not in the first place. Quite the contrary. Consider for example Holcombe's (2009) recent separation of two temporal limits of human visual system. On the one hand, there are fast limits (around $50 \mathrm{~ms}$ ) that are due to lowerlevel visual mechanisms such as first-order motion and binding local elements into global form. On the other hand, there are slow limits (around $200 \mathrm{~ms}$ ) that are due to high-level mechanisms such as word recognition, higher-order motion, and global form with color. As Holcombe (2009, p. 219) mentions, "[t] his notion of fast peripheral processing and slower central processing is an old one." What makes this separation significant for the topic at hand is that the threshold for flickering stimuli belongs to the first group consisting of lower-level mechanisms. The altered passage of time, however, is a general distortion affecting our perceptions as a whole (as reflected in the described phenomenology). Accordingly, there does not appear to be any reason to assume that temporal resolution in the early visual processes improves when the more central phenomenon of time slowing down occurs. This conclusion is emphasized by Stetson et al.'s claim that the subjective time is not a single unified entity, which means that subjective time is composed of subcomponents that can change independently of each other.

Second, if the temporal resolution of all of our visual perceptual processes were speeded up, then we should have more sensations, snapshots in Stetson et al.'s terminology, than we would have in normal situations. That is, sensations would follow each other faster than they normally do and in this sense we would see more during frightening events. Yet because the authors did not make any claims regarding the general improvement of our cognitive faculties, we would face more information than we can comfortably comprehend. Accordingly, rather than expecting the external world to appear to slow down, one would expect that in these kinds of situations time appears to go faster than normally. An analogous example for this would be modern (action) movies where the editing is done so that one scene is shown only for a brief moment. Here too, we are facing too much information and it is we who appear to be slow, trying to apprehend the images that follow each other too fast. Consequently, it appears that if the temporal resolution of perceptual processes were a factor in the experience of time slowing down, then contra Stetson et al., it should not improve but degrade when time slows down. As a result, even if we assume for the sake of argument that time slowed down for Stetson et al.'s subjects, it is only to be expected that the authors did not find any improvement in the temporal resolution during the fall.

One could obviously counter this objection by arguing that we have experiences of time slowing down because improvements in temporal resolution mirror the overall enhancement in neural processing, not only in the mechanisms of visual perception. Temporal resolution improvement understood in this way makes more sense: not only would our sensory systems provide us with more information but we would also be able to grasp this new information because our attention and other processes would be speeded up as well. While this is true, and a view resembling this will be sketched below, this does not help Stetson and his colleagues because the explanatory work in this alternative understanding of improved temporal resolution is done by our cognitive abilities - the capacity that determines whether we can grasp what we see faster than usual. This means that the temporal resolution of visual perception that Stetson et al. tested need not improve at all and for this reason the experiment was misguided. Given the problems with the rationale and methodology of the study and the results, it is clear that the improved temporal resolution for early visual perception cannot account for the experiences of time slowing down. Be that as it may, is it possible that a memory based account would fare better?

\section{EXPLANATION BASED ON MEMORY EFFECTS}

The memory account postulates that time appears to slow down because richer than usual memories are later erroneously interpreted to have spanned a greater period of time than the experience on which they are based actually did. To be fair to the authors it must be noted that they present this idea only briefly and in an almost sketch-like manner. However, the proposal is still worth elaborating on, as it can illustrate the consequences of not taking the reported phenomenology as a whole seriously.

To begin, the idea that our experience of time passing slowly would be due to richer memories and their possible secondary encoding is at odds with the fact that in Noyes and Kletti's (1976) study more than two thirds of the subjects reported an increased speed of thought. The increased speed of thought, if one reads the reports, can only refer to the idea that they had far more thoughts in a short time than they normally would - if our thoughts run faster than normally, then we can think more thoughts in that time than normally. The reason the memory account is at odds with the reports of an increased speed of thought is that because the real duration did not alter, the number of thoughts people have during the event would be the same regardless of the truth of memory account and hence the reports of an increased speed of thought remains unexplained. In fact, one could even argue that the situation is even worse because in these cases one should actually report that the speed of their thoughts was decreased (the number of thoughts remain the same but the subjects overestimate the duration of the event). Therefore, the phenomenology of increased speed of thought does not conform with the memory account.

It might be possible to resolve this discrepancy within the memory account by arguing that our memories of the event include many fabricated memories of thoughts. Although this possibility cannot be ruled out, it is still inadequate. First, while it is clear why memories would be particularly rich and strong (accidents are, after all, psychologically exceptional situations), this explanation does not provide any explanation for why such a massive fabrication of thoughts would take place - the reports of increased speed of thoughts are almost as common as reports of altered passage of time (68 vs. $75 \%$ according to Noyes and Kletti, 1976). Second, the 
idea of the fabrication of thoughts also fails to explain the fact that sometimes these thoughts have had causal relevance. Heim, for example, points out that under the conditions we are discussing here, people often "act on the basis of completely conscious, sustained, and complicated series of thoughts that are clear in every respect and often incredibly rapid (Noyes and Kletti, 1972, p. 48)." Their actions are not simple reflexes as the discussion in the second section illustrated. Given that in many cases people would not have survived if they had not acted purposefully, and that they could not have carried out such actions without an increased speed of thought, it appears likely that their speed of thought was indeed increased for a moment and the reports of it are not simply due to fabricated memories.

Another general shortcoming in Stetson and his colleagues' reasoning concerns the experience of time slowing down itself. As a sole explanation, the idea that time only appears to slow down because of unusually rich memories means that subjects never really experienced that time slowed down - it was all due to the retrospective judgment of the duration of the event. Yet when people report the mental state they had during accidents, it is the experiences of time slowing down that they report. Indeed, in this sense the memory account takes an antirealist position toward the reported phenomenology.

Bridging the gap from rich memories to experiences of time slowing down is a stretch, however, because when people make, to use Stetson et al.'s expression, an erroneous interpretation of the duration, the result is knowledge that time has slowed down, yet not an experience of it as one would have it. It is however the experiences of time slowing down that people report shortly after the event, and hence what is lacking here is an account how knowledge can turn into an experience of past episodes. For what it is worth, it can be asked further, what might be the evolutionary value of such mechanism that alters our memories after the life-threatening situations over the mechanism that speeds our thoughts and actions during those situations?

\section{HOW TO EXPLAIN THE EXPERIENCES DURING ACCIDENTS?}

The discussion of the previous two explanations for the reports of time slowing down emphasizes the fact that the reported phenomenology during frightening, sudden accidents as a whole needs to be taken seriously. Indeed, not only must one explain the phenomenology directly related to time slowing down (as the first explanation aimed to do) but also subjects' reports of vivid thoughts and unusually sharp vision and hearing (which the memory effects could partly explain). Nonetheless, neither explanation accounted for all the listed features of the distorted phenomenology.

The explanation for the altered temporal phenomenology that is argued for in this section accounts for the whole altered phenomenology and how some people can have acted fast and purposefully during the sudden, life-threatening situations. Arguably this has an advantage over the previously discussed proposal because it does justice to peoples' reports. The motivation of the explanation comes from Noyes and Kletti's idea that the experienced increased speed of thought and slowed down external time are interrelated the relation between the temporal properties of events in the external world and in internal states is distorted. As time in the real world does not really slow down during accidents, it is the internal processes that are somehow altered. Consequently, to go over the main points briefly, the explanation has two parts. First, a mechanism is suggested that explains how our cognitive processes could become faster during the sudden and frightening experiences. This will simultaneously account the non-temporal features of such experiences, such as altered attention and heightened perception. The previous does not yet explain the altered temporal phenomenology however. Even if our cognitive processes would be faster, that does not yet mean that our cognitive processes would appear to be unusually fast, or that we would have experiences of time slowing down and feelings that the event lasted longer than it actually did. Accordingly, the second part focuses on how our internal processes being faster can lead to such altered phenomenology in suitable situations.

To begin with, the mechanism that could account for our experiences during the sudden life-threatening situations needs to fulfill at minimum the following requirements: the mechanism needs to become active in such situations, it needs to activate fast, and it must have wide-ranging effects. The neuronal mechanism that fulfills these requirements is the locus coeruleus norepinephrine system, the main source of the neurotransmitter norepinephrine. The first thing to note about it is that it is (in addition to other things) part of a fight-or-flight response that humans and other primates have been postulated to exhibit. This response is triggered by a perception of a fearful and threatening situation, not threatening situations per se, and hence it is likely to play a role in the situations under discussion. Second, the phasic response (brief and high levels of discharge) of locus coeruleus norepinephrine system to highly salient as well as task-relevant stimuli occurs with a short latency of 100-150 ms and actually precedes behavior (Aston-Jones et al., 1994, 1997). Thus such activation is in accord with the fact that many accidents, like car accidents, last only a few seconds during which the effects could occur. Finally, given that noradrenergic neurons originating from locus coeruleus project exceptionally widely in the brain, the entire cerebral cortex for instance receives input from it, and that norepinephrine is one of the main neurotransmitters, it is unsurprising that the effects of released norepinephrine can be dramatic and wide-ranging (Berridge and Waterhouse, 2003).

As regards the function and effects of the activation of locus coeruleus and noradrenergic neurons that originate from it and release norepinephrine, converging evidence nowadays suggest that this system plays a complex role of "mediating shifts in attention and in promoting optimal behavioral performance" (Sara, 2009 , p. 220). In more detail, the most important effects of locus coeruleus norepinephrine system for the topic at hand are the following. First, the increase of norepinephrine has the result that our attention becomes more focused (Berridge and Waterhouse, 2003), the shifting of attention to new targets is facilitated (Aston-Jones and Cohen, 2005; Yu and Dayan, 2005), and the functional integration of brain systems related to attentional tasks improved (Coull et al., 1999). Second, the increase also facilitates our workingmemory performance (Arnsten et al., 1996; Ramos and Arnsten, 2007), task-related decision processes, and associated behavioral responses by increasing speed and accuracy of responses (Clayton et al., 2004; Aston-Jones and Cohen, 2005; Grefkes et al., 2010), and speeding up our behavioral adaptation to the environment 
(Devauges and Sara, 1990; Bouret and Sara, 2005). In addition, our clarity of thoughts possibly improves (Berridge and Waterhouse, 2003). Finally, with the increase of norepinephrine our sensitivity to sensory surrounds becomes enhanced and our perception is affected as neurons respond to stimuli faster and more accurately than in usual conditions (Berridge and Waterhouse, 2003; Aston-Jones and Cohen, 2005). Note that this does not mean that the temporal resolution of perception would improve, just that when a new stimulus appears, it is processed in less time than it would under normal situations. Altogether, the previous effects mean that the activation of locus coeruleus norepinephrine system speeds up our internal processes concerning the relevant features of the situation at hand and subsequently improves our behavioral responses to the environment.

In short, there is a well-established neurophysiological system that can be seen to play a role in the experiences that are under discussion. To begin with, its activity increases during the situations that are perceived to be threatening and it can exert its effects very rapidly. In addition, many of its known effects are in good accordance with the phenomenology discussed in the second section (namely heightened perception, altered attention). That is, this system provides a realist explanation for the non-temporal features of the phenomenology related to experiences we have been discussing. Given that it is plausible to think that such a system is in play here, there are good reasons to accept one of the consequences that this brings along: our cognitive processes, such as thinking and shift of attention, are facilitated and accordingly indeed our thought is faster during accidents where the system is activated. This is also in good accordance with the fact that the rate of our information processing can be speeded up by rather simple means. For example, the performance of participants is improved in many psychophysical experiments where the time is a crucial factor (such reporting as many letters as possible in a Sperling experiment) when this task is preceded by a train of sounds (clicks) for $5 \mathrm{~s}$ (Jones et al., 2011).

However, it is important to notice that even if our cognitive processes were faster, it does not yet mean that we have an experience that we are unusually fast. Likewise, although our duration estimations are likely to tend toward overestimation as a result of increased arousal, it does not follow yet that we have the phenomenology related to time slowing down. As an analogy, consider an amateur runner who is running on a new route and knows her heart rate. If the heart rate is exceptionally high, it could mean two things. On the one hand, she might be running exceptionally fast. On the other hand, she might be running roughly at her normal speed but she is not in the running condition she expected to be. That is, the high heart rate does not translate into the faster running speed without some additional information, such as the distance she has covered in certain time. Hence, what is missing is an explanation for why faster thought amounts to the experiences that we have ample time to perceive, attend, act, or whatever we are doing. Why do we feel ourselves being faster?

Theoretically it appears obvious that we cannot have an experience that we are somehow faster or slower if we do not have some kind of a criterion for normal "speed." Think, for instance, that one perceives for the first time a robot "dancing" (i.e., turning) champagne bottles in a winery. It is plausible to assume that in this case she does not have any idea of the pace at which this task is typically done, and accordingly it is impossible to say whether the robot does the task at normal speed or unusually fast or slow. The same holds for the experience that our thoughts are faster than usual. Without a background feeling about the "normal" rate of events unfolding in our awareness, we simply cannot know if the rate at which things unfold in a current situation is unusual or not. Indeed, the existence of some kind of criterion or background feeling of the normal speed appears theoretically necessary to explain the experiences we have been discussing.

The relevant question is, of course, what constitutes the sense of normal speed of things that we have been discussing. One suggestion can be found in William Goody's description of a patient, whose movements became unusually slow due to Parkinson's disease. After inquiring about the slowness of the patient's movements, Goody got the reply that "My own movements... seem normal unless I see how long they take by looking at a clock. The clock on the wall of the ward seems to be going exceptionally fast (as reported in Sacks, 2004)." While this quotation makes explicit the idea that we have a sense of the normal rate of passage of time, it also suggests that things external to us have an important role in determining the criterion - without the knowledge of the temporal relation between his movements and their real duration, the patient would not have had experiences of his movements being unusually slow.

Our speeded internal processes, due to an increased norepinephrine level, do not yet amount to the experience that we are faster - the speed of our thoughts can simply feel normal. However, when our actions are directed to the external world, they have the same kind of reference as Goody's patient had and we notice that we are unusually fast. For example, we might notice that we can shift our attention from one stimulus to another faster than normally. This means that things move less in the world during our shifts of attention than usual. Likewise, even if our actions themselves do not necessarily speed up much (after all, this is largely due to our muscles, not cortex), our actions could initiate unusually fast due to increased alertness and speed of decision making, both of which associated have been associated with higher levels of norepinephrine. In this situation, our sensory and re-afferent systems provide us with grounds to feel that somehow our internal processes are faster than usual. Indeed, it has been shown that the feedback of re-afferent systems plays an important role in determining the time in which we perceive our actions to occur (Obhi et al., 2009).

The support for the existence of the sense of normal speed that time passes is not only anecdotal, since our expectancies of the normal temporal course of our actions and their consequences become adapted to the prevailing circumstances. For example, when subjects press a key that results in a flash, and a short artificial fixed delay is introduced by the researchers, subjects adapt to this delay so that for them the flash appears at the same time as they press the key. This effect was most noticeable when the delay was removed (and the subjects have not adapted to the new situation yet), because in these situations subjects report that the flash appears before they press the key (Stetson et al., 2006).

If generalized, Stetson et al.'s "keypress-and-flash" experiment shows that we have (unconscious) expectations of the normal 
temporal relations between our decisions and actions (here pressing the key) and their consequences (flash occurs). Our motor, perception, and cognitive systems become adapted to a certain delay after which the consequences occur. This adaptation is influenced by the prevailing circumstances. The same thing can also be seen in crossmodal simultaneity tasks, where subjects adapt to the asynchrony of visual and auditory stimuli and thus do not perceive them being asynchronous anymore (e.g., Fujisaki et al., 2004; Zampini et al., 2005).

This example thus illustrates how there are expectations of the normal delay between our actions and their consequences, and suggests that there are similar expectancies pertaining other actions as well whether they are concrete or mental. Accordingly, we have some sense of how often and fast we can shift our attention from one thing to another, how fast we can react to things, and so forth. This idea combined with the assumption that our internal processes indeed are speeded brings about experiences that we are faster than usual. Furthermore, because the sense of rate at which time passes is determined by the speed of things in the external world as compared to the speed of our abilities to act according to those things, our feeling that we are somehow faster than usual also amounts to the experience that things in the external world happen slower than usual. That is, the temporal features of the phenomenology described in the section two can be accounted for.

One can object to this conclusion however by arguing that people do not have experiences of the sense of normal speed of things. While I agree that this is generally the case, it is important to notice that the feeling of normal speed at which things occur is something that need not be accessible to even the most perceptive introspection. Quite the contrary, it can be likened to the background feeling of ownership of our body parts or thoughts. This is not something that we normally feel, but when things go wrong, we do feel them as illustrated by clinical cases (such as somatoparaphrenia, see Vallar and Ronchi, 2009). Likewise, although we might not have an ever present feeling of the normal speed of things, something like that needs to be in the background for otherwise we could not notice when things are somehow unusual as in the case of "keypressand-flash" example or Goody's patient noticing how much time his movements take.

It is likewise unsuccessful to object to the current proposal based on the claim that it makes unreasonably strong predictions: when we drink coffee, our internal processes may become faster than before, but still we do not feel that external time has slowed down. The problem with this objection is that it ignores the details of "keypress-and-flash" experiment, which illustrates the importance of fast changes in the time course in which events unfold - because subjects adapt to the prevailing situations, we can only notice the changes that occur faster than we can adapt to the situation. When we drink coffee, the amount of caffeine in our system grows gradually and our expectancies of the temporal relations might change along with it. Hence, we do not have the experiences of us being faster and things moving in slow motion.

The provided explanation can also account for the fact people experience time slowing down only in sudden and frightening situations. First, the activity of a relevant fight-or-flight response only increases in threatening situations. Second, the change in the norepinephrine levels can either occur slowly or fast. Arguably the experimental situation in Stetson et al.'s (2007) study was not particularly fast - people had to put on the harnesses, get up to the tower where they were released, and wait for the release - and for this reason they had plenty of time to adapt to the likely increase in norepinephrine levels (and most probably also to increased adrenalin levels).

Finally, attentional effects and the increased speed of our cognitive processes suggest an alternative interpretation for the movie analogy that Stetson and his colleagues used. To begin with, given that attention is focused narrowly on the issues potentially relevant for survival, these things are perceived more clearly. Second, the shifts of attention are facilitated - we shift our attention from one thing to another unusually fast. When these shifts of attention are voluntary, they are known to cause the subjective expansion of time and sometimes even a feeling that time appears to stand still. Such feelings can occur in everyday situations when we, for example, turn our attention to the second hand of the clock and wait for it to jolt forward (Yarrow et al., 2001) or when, after dialing a telephone call and shifting attention shortly to something else, we turn attention back to the telephone call and wait to hear the next dialing tone (Hodinott-Hill et al., 2002). Combined these two suggest that, in the right conditions, one could have a succession of perceptions (following the shifts of our attention) where subjective time is expanded (and may even appear to stand still) and that these perceptions would be focused and well-scrutinized.

This line of reasoning lends itself to an interpretation of the movie analogy where subjects do not perceive more snapshots during the frightening events (as Stetson et al. hypothesized), but they have the feeling that the snapshots they perceive last longer than usual. It is not that there was an abundance of experiences, but abundance of time to scrutinize the perceptions. Hence this second interpretation proposes that there is no increase in the "refresh rate" of perception, but that we have the feeling of having more time to perceive things clearly than we normally do. Our enhanced cognitive processing, which facilitates our apprehension of things we perceive, also contributes toward having such feelings. Again analogy helps to understand this idea: when a film is seen in a very slow motion, we have an abundant time to perceive certain features in it. (For example, the question whether a galloping horse has all its feet off the ground at the same time could be determined only after Eadweard Muybridge produced a series of still images of such horse in 1870s.). It is this second interpretation that I think is the correct interpretation of the movie analogy.

\section{SUMMARY}

When people are suddenly faced with situations that they perceive as threatening, later they often report having had experiences with unusual phenomenology. I argue here that the phenomenology should be taken as given and reported in its entirety. This is because otherwise some of the most relevant phenomena in these situations-such as how people can act in unusually fast and purposeful ways to save their lives-would be left unaddressed. 
The provided explanation for the reported experiences can be summarized as follows: the experiences of time slowing down results from the fact that the relation between the temporal properties of events in the external world and internal states is suddenly (so that adaptation is yet to occur) distorted as result of increased speed of internal processes. In more detail, the explanation thus comes in two parts. First, it was suggested that cognitive processes are enhanced during the sudden and frightening situations - we are unusually fast. The explanation on how this could occur relied on the locus coeruleus norepinephrine system whose activity increases in these situations. Significantly, this increased activity correlates with enhanced cognitive processes and its effects match non-temporal features of the reported phenomenology (e.g., altered attention and heightened perception). Given that being faster does not equal the experience of being faster, this provided only a partial explanation of the experience however. The second part of the explanation for the experiences of time slowing down focused on how our internal processes being faster can lead

\section{REFERENCES}

Anderson, M., Reis-Costa, K., and Misanin, J. R. (2007). Effects of September 11th terrorism stress on estimated duration. Percept. Mot. Skills 104, 799-802.

Arnsten, A. F. T., Steere, J. C., and Hunt, R. D. (1996). The contribution of alpha 2-noradrenergic mechanisms of prefrontal cortical cognitive function. Potential significance for attention-deficit hyperactivity disorder. Arch. Gen. Psychiatry 53, 448-455.

Aston-Jones, G., and Cohen, J. (2005). An integrative theory of locus coeruleus-norepinephrine function: adaptive gain and optimal performance. Annu. Rev. Neurosci. 28, 403-450.

Aston-Jones, G., Rajkowski, J., and Kubiak, P. (1997). Conditioned responses of monkey locus coeruleus neurons anticipate acquisition of discriminative behavior in a vigilance task. Neuroscience 80, 697-715.

Aston-Jones, G., Rajkowski, J., Kubiak, P., and Alexinsky, T. (1994). Locus coeruleus neurons in monkey are selectively activated by attended cues in a vigilance task. J. Neurosci. 14, 4467-4480.

Bar-Haim, Y., Kerem, A., Lamy, D., and Zakay, D. (2010). When time slows down: the influence of threat on time perception in anxiety. Cogn. Emot. 24, 255-263.

Berridge, C. W., and Waterhouse, B. D. (2003). The locus coeruleusnoradrenergic system: modulation of behavioral state and statedependent cognitive processes. Brain Res. Rev. 42, 33-84.

Bouret, S., and Sara, S. J. (2005). Network reset: a simplified overarching theory of locus coeruleus noradrenaline function. Trends Neurosci. 28, 574-582.

Byram, S. (1997). Cognitive and motivational factors influencing time prediction. J. Exp. Psychol. Appl. 3, 216-239.

Campbell, L. A., and Bryant, R. A. novice skydivers. Behav. Res. Ther. 45, 1389-1392.

Clayton, E. C., Rajkowski, J., Cohen, J. D., and Aston-Jones, G. (2004). Phasic activation of monkey locus ceruleus neurons by simple decisions in a forced-choice task. J. Neurosci. 24, 9914-9920.

Coull, J., Büchel, C., Friston, K., and Frith, C. (1999). Noradrenergically mediated plasticity in a human attentional neuronal network. $\mathrm{Neu}$ roimage $10,705-715$.

Dainton, B. (2000). Stream of Consciousness. Philosophy. London: Routledge.

Devauges, V., and Sara, S. J. (1990). Activation of the noradrenergic system facilitates an attentional shift in the rat. Behav. Brain Res. 39, 19-28. and Nishida, S. (2004). Recalibration of audiovisual simultaneity. Nat. Neurosci. 7, 773-778.

Gibbon, J. (1977). Scalar expectancy theory and Weber's law in animal timing. Psychol. Rev. 84, 279-325.

Grefkes, C., Wang, L. E., Eickhoff, S. B., and Fink, G. R. (2010). Noradrenergic modulation of cortical networks engaged in visuomotor processing. Cereb. Cortex 20, 783-797.

Greyson, B., and Stevenson, I. (1980). The phenomenology of near-death experiences. Am. J. Psychiatry 137, 1193-1196.

Hodinott-Hill, I., Thilo, K., Cowey, A., and Walsh, V. (2002). (2007). How time flies: a study of

Fujisaki, W., Shimojo, S., Kashino, M.,

to an altered temporal phenomenology in suitable situations - how we can have the experience that we have ample time to perceive, attend, and act. This was explained by arguing that we have a sense of the normal speed of time passing and that this in turn depends on the temporal relation of our actions and external events. Our increased alertness, faster cognitive processes and initiation of actions make us aware that the relation is changed with the result that we have experiences with anomalous temporal characteristics.

\section{ACKNOWLEDGMENTS}

I would like to thank Christine Falter, Dan Lloyd, and David Eagleman for their comments on the earlier versions, and John Wearden and Marc Wittman for the discussions on the related issues. I am also very grateful the reviewers for their generous help and constructive comments. This work was supported by the Academy of Finland, a European Cooperation in Science and Technology (COST) action on Time in Mental Activity (TIMELY; TD0904), and Kordelin Foundation.

Auditory chronostasis: hanging on the telephone. Curr. Biol. 12, 1779-1781.

Holcombe, A. O. (2009). Seeing slow and seeing fast: two limits on perception. Trends Cogn. Sci. (Regul. Ed.) 13, 216-221.

Husserl, E. (1991). On the Phenomenology of the Consciousness of Internal Time (1893-1917). Dordrecht: Kluwer Academic.

James, W. (1890). The Principles of Psychology. New York: Dover.

Jones, L. A., Allely, C. S., and Wearden, J. H. (2011). Click trains and the rate of information processing: does "speeding up" subjective time make other psychological processes run faster? Q. J. Exp. Psychol. (Hove) 64, 363-380.

Lloyd, D. (2002). Functional MRI and the study of human consciousness. J. Cogn. Neurosci. 14, 818-831.

Loftus, E. F., Schooler, J. W., Boone, S. M., and Kline, D. (1987). Time went by so slowly: overestimation of event duration by males and females. Appl. Cogn. Psychol. 1, 3-13.

Noyes, R., and Kletti, R. (1972). The experience of dying from falls. Omega (Westport) 3, 45-52.

Noyes, R., and Kletti, R. (1976). Depersonalization in the face of lifethreatening danger: a description. Psychiatry 39, 19-27.

Noyes, R., and Kletti, R. (1977). Depersonilization in response to lifethreatening danger. Compr. Psychiatry 18, 375-384.

Obhi, S. S., Planetta, P. J., and Scantlebury, J. (2009). On the signals underlying conscious awareness of action. Cognition 110, 65-73.

Ramos, B. P., and Arnsten, A. F. T. (2007). Adrenergic pharmacology and cognition: focus on the prefrontal cortex. Pharmacol. Ther 113, 523-536.

Roy, M., Christenfeld, N., and McKenzie, C. (2005). Underestimating the duration of future events: memory incorrectly used or memory bias? Psychol. Bull. 131, 738-756.

Sacks, O. (2004). Speed: aberrations of time and movement. New Yorker 80, 60-69.

Sara, S. J. (2009). The locus coeruleus and noradrenergic modulation of cognition. Nat. Rev. Neurosci. 10, 211-223.

Stetson, C., Cui, X., Montague, P. R. and Eagleman, D. M. (2006). Motorsensory recalibration leads to an illusory reversal of action and sensation. Neuron 51, 651-659.

Stetson, C., Fiesta, M. P., and Eagleman, D. M. (2007). Does time really slow down during a frightening event? PLoS ONE 2, e1295. doi:10.1371/journal.pone.0001295

Treisman, M., Faulkner, A., Naish, P. L. N., and Brogan, D. (1990). The internal clock: evidence for a temporal oscillator underlying time perception with some estimates of its characteristic frequency. Perception 19, 705-743.

Vallar, G., and Ronchi, R. (2009). Somatoparaphrenia: a body delusion. A review of the neuropsychological literature. Exp. Brain Res. 192, 533-551.

Wearden, J. (1991). Do humans possess an internal clock with scalar timing properties? Learn. Motiv. 22, 59-83.

Wearden, J. (2008). The perception of time: basic research and some potential links to the study of language. Lang. Learn. 58(Suppl. s1), 149-171. 
Xuan, B., Zhang, D., He, S., and Chen, X. (2007). Larger stimuli are judged to last longer. J. Vis. 7, 1-5.

Yarrow, K., Haggard, P., Heal, R., Brown, P., and Rothwell, J. (2001). Illusory perceptions of space and time preserve cross-saccadic perceptual continuity. Nature 414, 302-304.

Yu, A. J., and Dayan, P. (2005). Uncertainty, neuromodulation, and attention. Neuron 46, 681-692.
Zäch, P., and Brugger, P. (2008). Subjective time in near and far representational space. Cogn. Behav. Neurol. 21, 8-13.

Zampini, M., Guest, S., and Shore, D. I. (2005). Audio-visual simultaneity judgments. Percept. Psychophys. 67, 531-544.

Conflict of Interest Statement: The author declares that the research was conducted in the absence of any commercial or financial relationships that could be construed as a potential conflict of interest.

Received: 06 October 2011; accepted: 26 May 2012; published online: 27 June 2012.

Citation: Arstila V (2012) Time slows down during accidents. Front. Psychology 3:196. doi: 10.3389/fpsyg.2012.00196
This article was submitted to Frontiers in Theoretical and Philosophical Psychology, a specialty of Frontiers in Psychology. Copyright (c) 2012 Arstila. This is an open-access article distributed under the terms of the Creative Commons Attribution Non Commercial License, which permits non-commercial use, distribution, and reproduction in other forums, provided the original authors and source are credited. 